\title{
Antagonistic actuation and stiffness control in soft inflatable robots
}

Kaspar Althoefer

Centre for Advanced Robotics, Faculty of Science and Engineering, Queen Mary University of London, Mile End Road, London E1 4NS, UK

k.althoefer@qmul.ac.uk

Soft robots promise solutions for a wide range of applications that cannot be achieved with traditional, rigid-component robots. A key challenge is the creation of robotic structures that can vary their stiffness at will, for example, by using antagonistic actuators, to optimize the interaction with the environment and to be able to exert high forces.

\section{From rigid to bioinspired soft robots}

Research into soft material robots has been driven by the interest to create robotic structures that can better adapt to their environment than their traditional counterparts made from rigid bodies, and that can conduct manipulation tasks whilst reducing the computational burden of control and motion planning ${ }^{1}$. With their heavy and rigid manipulation arms, oldgeneration robots may have helped to revolutionize the manufacturing industry, but have long been plagued by the inherent inability to compliantly adapt to their environment. Advances in force and torque sensing and the development of sophisticated control strategies have enabled rigid robotic systems to behave in a compliant manner suitable for safe humanrobot interaction. However, soft material robots represent a drastic departure from the common industrial robot. Soft robots are capable of environment-responsive manipulation and locomotion and they can operate without the need for skeletal structures ${ }^{1}$.

Scientists are looking for inspiration in nature for the design of soft robots. Octopuses with their dexterous arms, elephants with their flexible trunks and chameleons with their extendable tongues provided natural models for the first generation of soft robots whose bodies' interaction with the environment impacts on and even defines their behavior [Nakajima]. Increased interest in the use of soft, compliant materials for the creation of highly dexterous robots ${ }^{2,3}$ is evidenced by the surge in publications in monothematic journals, such 
as Soft Robotics (published by Mary Ann Liebert, Inc.), and by the initiation of dedicated soft robotics sessions at major robotics conferences, such as the International Conference on Robotics and Automation (ICRA) and Intelligent Robotics and Systems (IROS). Numerous proposals for novel flexible robots, based on rigid, soft and hybrid materials, are continuously emerging [Stilli, Kier]. It has been recognized that their boneless soft structures makes them well-suited for close human-robot interaction and the handling of fragile objects [Deimel].

Continuum hyper-redundant designs are investigated for the creation of soft robots for applications in minimally invasive surgery, rehabilitation, assistive healthcare technologies, disaster scenarios and underwater exploration. Roboticists contend that soft robotic technologies will become indispensable for safe human-robot interaction in manufacturing and thus will play a key role in the $4^{\text {th }}$ industrial revolution.

\section{Antagonistic actuation in soft robots}

Soft robots possess better morphological capabilities than traditional rigid-link robots owing to their compliant nature; however, exerting high forces for object manipulation and task handling, often involving high payloads ${ }^{2}$, remains a challenge, especially for robots made from rubber-like silicone. Therefore, the creation of robots that can adjust their stiffness constitutes a key milestone in the soft robotics field.

Antagonistically arranged muscles allow animals and humans to adjust the stiffness of their limbs to achieve sophisticated motion trajectories and precise control of interaction forces when handling objects; for example, humans can easily pick up fragile objects, such as eggs, without damaging them and, when required, actively apply the right amount of force to crack them open.

Antagonistic actuation in robots can be realized by two or more actuators that antagonistically act on a joint in a rigid-component arm, enabling the movement of the attached link and stiffness control of the joint. Such systems rely on a rigid bone-like structure to relay and amplify the input forces of the actuator through rigid links ${ }^{3}$. However, in nature, high dexterity and stiffness control can be achieved in actuated appendages without the need for an underlying skeletal structure. For example, the octopus is a mollusk, which can move its flexible arms without a bone in its body. Each octopus arm can be individually controlled 
to follow motion trajectories, can be squeezed to fit through narrow passages and can stiffen to conduct tasks such as catching pray or walking on the seabed ${ }^{3,4}$. Octopus arms are predominantly composed of 3D arranged muscle groups that can operate in longitudinal, transversal and oblique mode. The muscles can contract, elongate, bend and twist by controlling the interplay of the perpendicularly arranged muscle groups, and they can actively adjust their stiffness ${ }^{3,4}$. A layer of connective tissue surrounds the central nerve bundle and a second layer, which extends along the whole arm, coats the muscle groups and thus, provides the origin of and insertion points for the muscle fibers (FIG. 1).

The muscular structure and function of the octopus can be recreated by designing inflatable robotic structures made of soft, but inextensible textile materials, integrating pneumatic and tendon-based actuation in an antagonistic way ${ }^{2,5}$. This type of robot consists of an outer cylindrical sleeve made of an airtight, inextensible fabric, forming a chamber, and tendons originating from various points along the sleeve. Pressurizing the chamber triggers the robotic structure to emerge and the attached tendons can be pulled to bend the structure into different directions. These robotic structures can adjust their stiffness and geometry to adapt to their environment, that is, adapt a flexible state to squeeze through narrow openings and become stiffer to handle high payloads. Mimicking the arm stiffening of an octopus, the inflatable robot arm exploits the opposing forces of the tendons (pulling) and pressurized air (pushing outwards longitudinally and laterally) in unison with the outer fabric (resembling the role of the connective tissue), to simultaneously achieve a certain stiffness and perform a specific motion (FIG. 1). To the best knowledge of the author, this is the first approach to design an actuated robotic structure that can change its state from entirely collapsed and foldable to a state of high stiffness.

\section{Outlook}

Robots have evolved from mechanized giants with rigid links to small, flexible and soft structures. Soft robotics is based on the fusion of different technologies.

\section{Acknowledgments}


The work described in this paper was partially funded by the Seventh Framework Programme of the European Commission under grant agreement 287728 in the framework of EU project STIFF-FLOP.

\section{References}

1. Kohei Nakajima, Helmut Hauser, Rongjie Kang, Emanuele Guglielmino, Darwin G. Caldwell and Rolf Pfeifer, A soft body as a reservoir: case studies in a dynamic model of octopus-inspired soft robotic arm, COMPUTATIONAL NEUROSCIENCE, 09 July 2013, doi: $10.3389 /$ fncom.2013.00091

2. Agostino Stilli, Helge A Wurdemann and Kaspar Althoefer, Shrinkable, stiffnesscontrollable soft manipulator based on a bio-inspired antagonistic actuation principle, 2014 IEEE/RSJ International Conference on Intelligent Robots and Systems (IROS 2014), Pages 2476-2481, 2014/9/14

3. William M. Kier; The Musculature of Coleoid Cephalopod Arms and Tentacles, Frontiers: Cell and Developmental Biology, Review Article, 18 February 2016; doi.org/10.3389/fcell.2016.00010

4. Rongjie Kang, David T Branson, Tianjiang Zheng, Emanuele Guglielmino and Darwin G Caldwell, Design, modeling and control of a pneumatically actuated manipulator inspired by biological continuum structures, Bioinspir. Biomim. 8 (2013), doi:10.1088/1748-3182/8/3/036008

5. Farahnaz Maghooa, Agostino Stilli, Yohan Noh, Kaspar Althoefer, and Helge A Wurdemann, Tendon and pressure actuation for a bio-inspired manipulator based on an antagonistic principle, 2015 IEEE International Conference on Robotics and Automation (ICRA) Washington State Convention Center Seattle, Washington, May 26-30, 2015, Pages 2556-2561, 2015/5/26 


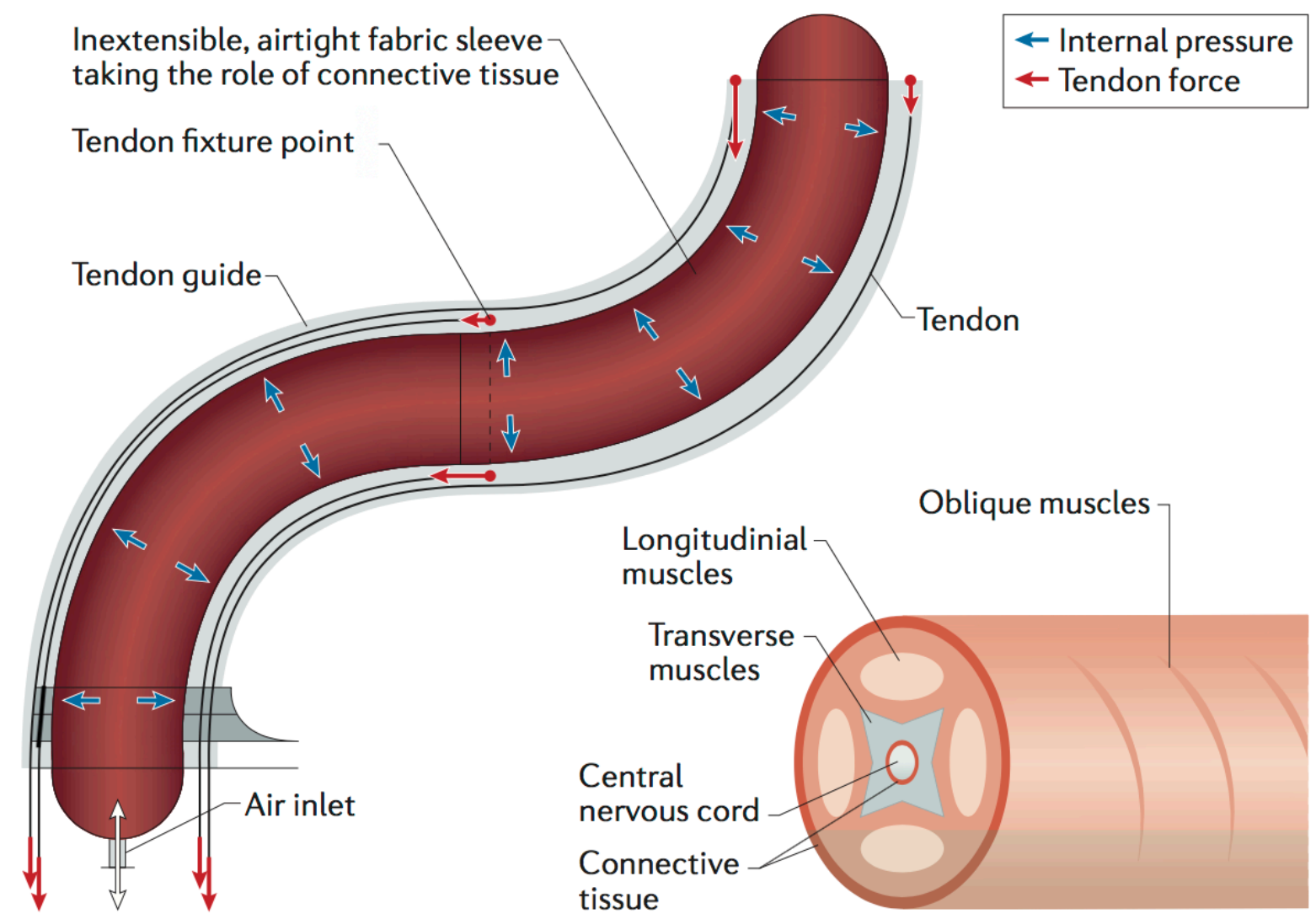

Figure 1. Octopus-inspired robot. a) Muscular arrangement in an octopus arm. The muscles are organized in three groups: transverse, longitudinal and oblique muscles. Connective tissue surrounds the central nervous cord and the outer layer of the arm. The arm stiffens by activating all three muscle groups within the two connective tissue layers. b) The octopusinspired inflatable robot consists of an inextensible sleeve that resembles the role of the connective tissue. Antagonistic pneumatic and tendon-based actuation enable the robot to navigate and to adapt its stiffness. Panel $a$ is adapted from REF. 4, with permission from IOP Publishing. Panel $b$ is adapted from REF. 2, with permission from IEEE. 